\title{
Diagnostic and treatment delays in breast cancer in association with multiple factors in Pakistan
}

\author{
Imran Majeed,,$^{1,3}$ Rana Ammanuallah, ${ }^{2}$ Abdul Wahed Anwar, ${ }^{3}$ Hafiz M. Rafique ${ }^{2}$ and Faiza Imran ${ }^{1}$
}

${ }^{1}$ Radiation Oncology Department, Jinnah Hospital, Allam Iqbal Medical College, Lahore, Pakistan. (Correspondence to: Imran Majeed: bisimran@gmail. com). ${ }^{2}$ Physics Department, Punjab University, Lahore, Pakistan. ${ }^{3}$ Physics Department, University of Engineering and Technology, Lahore, Pakistan.

\begin{abstract}
Background: Breast cancer has the highest incidence rate among all types of cancer worldwide. There is strong evidence that delay in presentation to an oncologist may lead to a decrease in survival.

Aims: This study explores factors causing diagnostic and treatment delays among the breast cancer patients enrolled in Jinnah Hospital, Lahore, from 2016 to 2018.

Methods: Data from 372 patients were collected, including tumour characteristics, first symptoms, knowledge and experience of breast cancer, first visit to a doctor, etc. We calculated the patient, physician, treatment, system and total delay intervals.

Results: Breast cancer cases showed longer mean patient delay in older women (> 50 years) in comparison with younger women. Women with painless lump as the initial symptom showed the longest delay with median total delay 280 days (25th and 75th percentiles 140 and 410 days respectively). Initial symptoms were correlated with total delay $(P=0.036)$. Educated women showed shorter delay in treatment compared with illiterate women $(P=0.068)$. Rural residence showed significant delay $(P=0.007)$. Lump size showed correlation with delay $(P=0.039)$. Patients with low household income $(<$ Rs 10 000) had greater delay in diagnosis $(P=0.027)$ and actively employed women showed shorter delay $(P<0.0001)$. Unmarried women were diagnosed earlier than married $(P<0.001)$.
\end{abstract}

Conclusions: Women showed delay in presentation due to lack of resources and lack of awareness about the disease. They presented late due to fear of surgery and chemotherapy. Using traditional treatment methods leads to diagnosis of the disease at more advanced stages.

Keywords: breast cancer, time delays, socioeconomic factors, Pakistan

Citation: Majeed I; Ammanuallah R; Anwar A; Rafique H; Imran F. Diagnostic and treatment delays in breast cancer in association with multiple factors in Pakistan. East Mediterr Health J. 2021;27(1):23-32. https://doi.org/10.26719/emhj.20.051

Received: 01/05/19; accepted: 08/12/19

Copyright (C) World Health Organization (WHO) 2021. Open Access. Some rights reserved. This work is available under the CC BY-NC-SA 3.0 IGO license (https://creativecommons.org/licenses/by-nc-sa/3.o/igo).

\section{Introduction}

Breast cancer has the highest incidence rate among all types of cancer worldwide (1): the age standardized rate per 100000 for breast cancer diagnosis was 46.3 in 2018. The rate for the developed countries was reported to be 75.2 in comparison to 32.8 for the developing countries. The International Agency for Research on Cancer reported breast cancer as the second most common cancer, with $11.6 \%$ of the total 18.1 million cancer cases in 2018 (2). Cancer is the leading barrier to increased life expectancy in the 21st century. There is strong evidence that delay in presentation to an oncologist may lead to a decrease in survival (3). These results highlight the need for research regarding awareness of the early warning signs of breast cancer in Pakistani women (4). Most breast cancers begin in the breast lobules or in the ducts of the breast, for which the most common sign is a painless lump (5). Breast cancer mortality can be reduced with early detection and improvements in the health care system (6).

The worldwide survival rates for breast cancer exhibit large variations across the globe from the developed to the developing countries. Indicators of 5-year survival rates from the developed part of the world include: America (83.2\%), Australia (80.7\%), Japan (81.6\%) and Sweden (80.0\%). In the developing countries, Algeria (38.8\%) Brazil (58\%), Gambia (12\%) and India (52.1\%) have noticeably low survival rates among diagnosed cases (7).

Delay is found between the the appearance of the first symptoms and time of diagnosis and initiation of treatment in women who have breast cancer. Early diagnosis and treatment within 30 days is beneficial for patients and helps to increase survival rates (8). Delay between the appearance of symptoms and presentation to an oncologist depends on the patient's behaviour and beliefs (9).

Long waiting times can lead to advancement and complications in the disease process. Li et al. studied Chinese breast cancer patients and associated the longer detection to treatment time interval with rural residence, low education level and older age (10). Nonavailability of early detection programmes increases delay in the detection of breast cancer at a curable stage. Pakistani oncologists follow the TNM staging developed by American Joint Committee on Cancer. The stage at 
diagnosis indicates the expected prognosis. In a recent study in Pakistan, Gulzar et al. reported that $88.8 \%$ of breast cancer patients were diagnosed late and 59.0\% at an advanced stage (11). The study associates $81.1 \%$ of delays to ignorance, painless lump and scarce financial resources. Lopes et al. in Brazil observed 102 days delay in diagnosis and 57 days delay in treatment of breast cancer (12). The study described low education as a main cause for late diagnosis. A study from Mali observed patient interval to report breast cancer of 4.8 months, diagnostic interval 0.9 months and treatment interval 1.3 months (13). They concluded that working women living in capital had shorter delay time in comparison to housewives and those living in rural areas. Maghous et al. reported delay in $70.1 \%$ patients due to personal reasons and $13.9 \%$ due to medical facility reason for Moroccan women (14). They recommended the training of medical practitioners and improving the awareness of general public about breast cancer to early detection of cancer. Jaiswal et al. found presentation to diagnostic time interval of 23 days and other treatment times within the published limits except time for radiotherapy at Denver hospital Colorado (15). They associated longer than median time intervals with ethnicity, language, stage, method of presentation and surgical treatment.

There are multiple factors to be focused on including growth rate of tumour, misunderstandings about cancer, age at detection, awareness in women and heath policy while addressing the core issue of early cancer detection. Breast cancer treatment has several sequential processes: surgery, chemotherapy and radiation therapy. Traditionally in Pakistan, mammography and biopsy are the choices for detection. Long waiting times in the diagnosis process can be reduced to increase patient comfort. Biopsy, consultation, surgical and patientperceived wait times are some factors which increase the system delay in breast cancer treatment (16). Women with family history of breast cancer had greater awareness of breast cancer and experienced greater fear about the symptoms in comparison with women without any family history (17).

The main objectives of this study were to determine the number of women with breast cancer who experienced delay in diagnosis and to determine causes of late presentation and the factors that contribute to system delay and overall wait time at the beginning of treatment among breast cancer patients enrolled at Jinnah Hospital, Lahore during 2016-2018 using quantitative strategies.

\section{Methods}

\section{Ethics}

This study was carried out at Jinnah Hospital, Lahore, and was approved by the hospital ethical committee. The questionnaire and consent forms were designed in the local language, Urdu, for better communication. The consent form was prepared according to the guidelines of the ethical committee. The guidelines of the Helsinki Declaration were followed in conducting this research work.
The consent forms were signed by the participants to accept the use of their medical records and interview data.

\section{Participants}

Data were collected during the 2 years January 2016-January 2018. Over this period, a total of 428 breast cancer patients were enrolled in Jinnah Hospital, Lahore. Those women who met the following criteria were included in the research:

\section{- diagnosed case of biopsy-proven breast cancer}

- understand Urdu

- visited Allam Iqbal Medical College/Jinnah Hospital for treatment

- complete records were available for them

- agreed to provide consent for participation in research.

Total population sampling allowed deep insight to study the factors involved in delay for the treatment and diagnosis of cancer. This is a method through which we included all the patients fulfilling our criteria and excluded those who did not meet the criteria. Total population sampling decreases the guesswork in research and provides a complete picture of the factors causing delays. From the 428 breast cancer patients attending during the study period, 372 fulfilled our inclusion criteria and the remaining 56 patients were excluded: 7 with benign lumps, 11 for noncooperative behaviour, 5 who had other cancers, 7 who were unable to answer the questions (for any reason), 6 who were mentally not able, 8 who did not agree to sign the consent form and 12 for whom information was missing on initial breast symptom and dates. The majority of related work utilized the whole population as the sample (12-14,18).

Women with self-detected or imaging-detected (mammogram or MRI) symptoms were included. All the patients were informed about the purpose of the study. We identified patients and took their medical history from the hospital medical records along with the date of admission and the date of surgery. When the patients were unable to provide the date of initiation of their symptoms, they were asked to provide the month and then the date was estimated as 15th of that month. The date of first consultation with a doctor was obtained from the receipt provided by the doctor to the patient. Each interview took 15-25 minutes but some took longer due to illiteracy and a socially complex environment. The interviews were conducted independently by trained staff. Information was collected about patient's tumour characteristics, first symptoms, experiences with a breast problem, their knowledge about breast cancer, first visit to a doctor, number of health care facility visits before the diagnosis, sociodemographic information, age, marital status, residential status, education, profession, monthly household income, comorbidities, tumour stage, history of breast disease and family history of breast cancer. We used the strategy to define the stage of the disease, I, II, III and IV. The patients were divided into 5 groups according to age (21-30, 31-40, years, etc.). Data on education level, 
residential status and monthly household income were also collected.

\section{Data}

We categorized the delay into 4 types: patient delay, physician delay, system delay and treatment delay. Patient delay was defined as the time from the first appearance of symptoms to the first visit to a hospital. Physician delay was defined as the time from first consultation to diagnosis of the disease. Referral delay (period between first consultations with the health care provider to first referral to hospital) was considered a part of physician delay. The time from biopsy to surgery, from surgery to onset of chemotherapy or from biopsy to onset of chemotherapy and time from surgery to onset of radiotherapy were defined as system delay. Treatment delay comprised the time between the diagnosis of cancer to the start of treatment.

Questions were asked of the patients to determine the causes of late presentation such as: Were you using alternative/traditional medicine? Did you not have enough money to pay the hospital bills? Did you present late due to the lump being small and painless? We calculated the patient delay, physician delay, treatment delay, system delay and total delay intervals and determined the median and 25th and 75th percentiles among different groups of patients for the comparison of multiple factors. The percentile methods help in the comparison of health indicators in the environment.

\section{Statistics}

We used SPSS software for statistical analysis. The Pearson correlation and Chi-squared were used to determine the relationships between variables. Significance was set as $P<0.05$.

\section{Results}

\section{Delay}

Characteristics of the participants are illustrated in Table 1. A total of 372 women were interviewed; all were residents of Punjab province; 81.7 were married. Age at the time of diagnosis was $20-68$ years, $33.0 \%$ were in the range 41-50 years. Half the women reported painless lump as the initial symptom.

Table 2 shows patient delay, referral delay and oncologist delay. Different age groups show different patient delay, the shortest patient delay, median 40 days, was found in young women (11-20 years) and the longest patient delay, median 152 days was found in the age group from 51-60 years. Women with painless lump as their initial symptom showed the longest delay with median total delay 280 days. Lump size is also a significant factor in association with delay, patients with lump size $1-5 \mathrm{~mm}$ presented with median 244 days total delay, $Q_{25}$ (136 days) and $\mathrm{Q}_{75}$ (382 days), longer than patients with lump size $>5 \mathrm{~mm}$.

\begin{tabular}{|c|c|}
\hline Factors & No. (\%) \\
\hline \multicolumn{2}{|l|}{ Age at diagnosis (years) } \\
\hline $11-20$ & $18(4.8)$ \\
\hline $21-30$ & $56(15.0)$ \\
\hline $31-40$ & $84(22.5)$ \\
\hline $41-50$ & $123(33.0)$ \\
\hline $51-60$ & $62(16.6)$ \\
\hline $61-70$ & $29(7.8)$ \\
\hline \multicolumn{2}{|l|}{ Initial symptoms } \\
\hline Painless lump/tumour & $189(50.0)$ \\
\hline Change in breast & $48(13.0)$ \\
\hline Discharge/bleeding & $33(8.9)$ \\
\hline Lump under arm & $47(12.6)$ \\
\hline Dimpling & $23(6.1)$ \\
\hline Rash & $32(8.6)$ \\
\hline \multicolumn{2}{|l|}{ Cancer detection } \\
\hline Self-detected & $143(38.4)$ \\
\hline Exam detected & $91(24.5)$ \\
\hline $\begin{array}{l}\text { Imaging detected (mammogram or magnetic } \\
\text { resonance imaging) }\end{array}$ & $138(37.1)$ \\
\hline \multicolumn{2}{|l|}{ Education } \\
\hline Illiterate & $162(43.5)$ \\
\hline Primary & $43(11.5)$ \\
\hline Middle & $22(6.0)$ \\
\hline Metric & $72(19.3)$ \\
\hline Intermediate & $34(9.1)$ \\
\hline Bachelor & $23(6.1)$ \\
\hline Masters & $16(4.3)$ \\
\hline \multicolumn{2}{|l|}{ Family history of breast cancer } \\
\hline Yes & $64(17.2)$ \\
\hline No & $308(82.8)$ \\
\hline \multicolumn{2}{|l|}{ Active employment } \\
\hline Yes & $87(23.3)$ \\
\hline No & $285(76.7)$ \\
\hline \multicolumn{2}{|l|}{ Monthly household income (Pakistan rupees) ${ }^{a}$} \\
\hline$<10000$ & $84(22.5)$ \\
\hline $10000-20000$ & $62(16.6)$ \\
\hline $21000-30000$ & $65(17.4)$ \\
\hline $31000-40000$ & $78(21.0)$ \\
\hline $41000-50000$ & $53(14.2)$ \\
\hline$>50000$ & $30(8.0)$ \\
\hline \multicolumn{2}{|l|}{ Marital status } \\
\hline Married & $304(81.7)$ \\
\hline Unmarried & $68(18.3)$ \\
\hline \multicolumn{2}{|l|}{ Lump size (mm) } \\
\hline $1-5$ & $164(44.0)$ \\
\hline $6-10$ & $83(22.3)$ \\
\hline $11-20$ & $81(21.7)$ \\
\hline $21-30$ & $26(6.9)$ \\
\hline $31-50$ & $18(4.8)$ \\
\hline
\end{tabular}




\begin{tabular}{|c|c|}
\hline Factors & No. (\%) \\
\hline \multicolumn{2}{|l|}{ Tumour stage on diagnosis } \\
\hline Stage I & $75(20.1)$ \\
\hline Stage II & $128(34.4)$ \\
\hline Stage III & $102(27.4)$ \\
\hline Stage IV & $67(18.0)$ \\
\hline \multicolumn{2}{|l|}{ First start self-treatment } \\
\hline Yes & $132(35.5)$ \\
\hline No & $240(64.5)$ \\
\hline \multicolumn{2}{|l|}{ Place of residence } \\
\hline Village & $103(27.7)$ \\
\hline Town & $93(25.0)$ \\
\hline Tehsil & $57(15.3)$ \\
\hline District & $68(18.2)$ \\
\hline Division & $51(13.7)$ \\
\hline \multicolumn{2}{|l|}{ Menopausal status } \\
\hline Premenopausal & $128(34.4)$ \\
\hline Postmenopausal & $244(65.6)$ \\
\hline \multicolumn{2}{|l|}{ Comorbid conditions } \\
\hline None & $257(69.0)$ \\
\hline$\geq 1$ & $115(31.0)$ \\
\hline \multicolumn{2}{|l|}{ First consultation } \\
\hline Health worker or nurse & $142(38.1)$ \\
\hline Doctor clinic & $124(33.3)$ \\
\hline Private hospital & $34(9.1)$ \\
\hline Government hospital & $72(19.2)$ \\
\hline
\end{tabular}

$a_{1}$ US $\$=139.9$ Rs.

A difference was found between married and unmarried women in regard to in total delay. The unmarried women showed short patient delay compared with married women, however physician and system delay were almost the same. Educated women showed a shorter delay in treatment compared with illiterate women. Working women faced shorter physician and system delay. Women who were dependent on their family showed longer patient delay compared with those who were independent.

We combined the referral and oncologist delay into a single term physician delay (Table 3). System delay and treatment delay are also shown in Table 3. There was a significant difference in total delay time among the women according to their area of residence. Patients from villages and small towns showed longer patient delay and faced longer physician and system delay compared with those who lived in cities (Tables 2,3). Women from villages also showed total delay higher than in cities with median 276 days; 25 th and 75th percentiles were 163 and 370 days respectively, (Table 4). Household income was an important factor.

Patients with low household income ( $<10$ ooo rupees) showed longer delays in diagnosis and treatment (Table 3); we found that most of these patients had started treatment with nonmedical traditional methods. Patients with household income $>50000$ rupees faced shorter physician and system delay and had a shorter total delay in treatment (Table 4).

\section{Factors associated with delay}

The association between multiple factors and delays was calculated using the Pearson correlation ( $r$ ). For initial symptoms, $r=0.847(P=0.036)$, i.e. there is a strong association between delay and symptoms initially noticed (Table 5). Residence was also associated with delays: $r=$ $0.965(P=0.007)$. There was a correlation between monthly income and delay $(r=0.86, P=0.027)$. Delay has a correlation $r=0.896$ with lump size $(P=0.039)$. Education was also correlated with delay $(r=0.7195)$, but this was not statistically significant $(P=0.068)$. Age was not strongly associated with delay $(P=0.322)$.

Chi-squared test results associating total delay with active employment, menopause status and marital status are shown in Table 6. For marital status chi-squared was 49.917, and this was statistically significant $(P<0.001)$. Actively employed women had significantly shorter delay times: chi-squared was $21.588(P<0.001)$. For menopause status, chi-squared was 2.731 , but this was not statistically significant $(P=0.098)$.

\section{Discussion}

This research work has revealed that women with breast cancer in Pakistan presented with significant delays, which leads to a high mortality rate. Although it is important to research delays in breast cancer patients, limited related studies are conducted in Pakistan. Our research reported $65 \%$ patients had a delay of more than median 90 days in Lahore in comparison with a study conducted by Habibullah et al. in Karachi with 50\% women (19). Gulzar et al. reported $92.8 \%$ of patients with initial symptom of painless lump in comparison to $50 \%$ in our study (11); painless lump was an important factor for delay in both studies. The common causes reported in patients in previous studies were lack of awareness and lack of knowledge related to breast cancer. This leads to diagnosis at later stages and consequently to lower survival rates. Grossie et al. in a study in Mali reported median time to visit first medical advice was 144 days (13). Kitano et al. researched treatment delay among $18 \%$ of breast cancer patients in Tokyo (20).

Our study has highlighted multiple factors responsible for the delays in breast cancer patients at Lahore, Pakistan. Multiple causes are recognized in the process of diagnosis and treatment of breast cancer. The women considered their breast changes temporary and harmless, in addition painless lump made them feel relaxed. The general public remains ignorant about these important signs and symptoms of breast cancer, which can lead to death due to negligence. The low literacy among our sample lead to ignorance and caused the other factors of delay to emerge. The cultural beliefs made it difficult for the population to realize the importance of women's education. This study emphasizes the fact that 


\begin{tabular}{|c|c|c|c|c|c|c|c|c|c|c|}
\hline \multirow[t]{2}{*}{ Characteristic } & \multirow[t]{2}{*}{ No. } & \multicolumn{3}{|c|}{ Patient delay (days) } & \multicolumn{3}{|c|}{ Referral delay (days) } & \multicolumn{3}{|c|}{ Oncologist delay (days) } \\
\hline & & Median & 25th & 75th & Median & 25th & 75th & Median & 25th & 75th \\
\hline Overall & 372 & 255 & 120 & 345 & 16 & 7 & 24 & 7 & 4 & 14 \\
\hline \multicolumn{11}{|l|}{ Age (years) } \\
\hline $11-20$ & 18 & 40 & 24 & 70 & 10 & 6 & 18 & 5 & 3 & 11 \\
\hline $21-30$ & 56 & 60 & 30 & 165 & 12 & 7 & 21 & 4 & 2 & 9 \\
\hline $31-40$ & 84 & 126 & 45 & 216 & 16 & 9 & 30 & 6 & 3 & 12 \\
\hline $41-50$ & 123 & 115 & 64 & 184 & 15 & 9 & 32 & 5 & 2 & 10 \\
\hline $51-60$ & 62 & 152 & 90 & 224 & 12 & 5 & 24 & 8 & 5 & 14 \\
\hline $61-70$ & 29 & 146 & 84 & 173 & 20 & 11 & 36 & 7 & 3 & 15 \\
\hline \multicolumn{11}{|l|}{ Initial symptoms } \\
\hline Painless lump/tumour & 189 & 180 & 105 & 325 & 24 & 8 & 38 & 6 & 2 & 12 \\
\hline Change in breast & 48 & 124 & 63 & 186 & 16 & 5 & 24 & 7 & 4 & 16 \\
\hline Discharge/bleeding & 33 & 135 & 74 & 215 & 14 & 8 & 25 & 8 & 3 & 16 \\
\hline Lump under arm & 47 & 90 & 45 & 120 & 15 & 6 & 28 & 6 & 2 & 10 \\
\hline Dimpling & 23 & 135 & 85 & 180 & 9 & 4 & 21 & 5 & 2 & 9 \\
\hline Rash & 32 & 75 & 30 & 105 & 12 & 7 & 25 & 8 & 3 & 14 \\
\hline \multicolumn{11}{|l|}{ Menopausal status } \\
\hline Premenopausal & 158 & 135 & 60 & 205 & 18 & 7 & 28 & 9 & 4 & 16 \\
\hline Postmenopausal & 214 & 165 & 90 & 255 & 16 & 5 & 24 & 12 & 5 & 20 \\
\hline \multicolumn{11}{|l|}{ Marital status } \\
\hline Married & 304 & 225 & 145 & 300 & 18 & 12 & 30 & 8 & 3 & 15 \\
\hline Unmarried & 68 & 180 & 105 & 230 & 15 & 8 & 21 & 7 & 2 & 11 \\
\hline \multicolumn{11}{|l|}{ Education } \\
\hline Illiterate & 162 & 240 & 135 & 320 & 14 & 5 & 22 & 9 & 4 & 16 \\
\hline Primary & 43 & 225 & 120 & 270 & 15 & 6 & 28 & 7 & 3 & 12 \\
\hline Middle & 22 & 165 & 75 & 210 & 12 & 5 & 20 & 8 & 5 & 14 \\
\hline Metric & 72 & 180 & 90 & 240 & 16 & 9 & 30 & 7 & 4 & 15 \\
\hline Intermediate & 34 & 115 & 60 & 195 & 18 & 10 & 28 & 6 & 2 & 12 \\
\hline Bachelor & 23 & 60 & 25 & 135 & 14 & 5 & 24 & 8 & 3 & 16 \\
\hline Masters & 16 & 45 & 30 & 90 & 11 & 6 & 20 & 5 & 2 & 10 \\
\hline \multicolumn{11}{|c|}{ Household income (Rs/month) ${ }^{a}$} \\
\hline$<10000$ & 84 & 270 & 135 & 330 & 22 & 12 & 38 & 8 & 4 & 16 \\
\hline $10000-20000$ & 62 & 240 & 120 & 315 & 20 & 9 & 36 & 8 & 3 & 14 \\
\hline $21000-30000$ & 65 & 195 & 105 & 280 & 12 & 7 & 24 & 6 & 2 & 10 \\
\hline $31000-40000$ & 78 & 165 & 90 & 225 & 9 & 4 & 20 & 9 & 4 & 14 \\
\hline $41000-50000$ & 53 & 120 & 90 & 180 & 11 & 5 & 25 & 7 & 4 & 12 \\
\hline$>50000$ & 30 & 75 & 30 & 105 & 8 & 3 & 15 & 6 & 3 & 10 \\
\hline \multicolumn{11}{|l|}{ Area of residence } \\
\hline Village & 103 & 285 & 150 & 360 & 25 & 10 & 40 & 10 & 4 & 18 \\
\hline Town & 93 & 225 & 120 & 320 & 18 & 7 & 30 & 10 & 6 & 15 \\
\hline Tehsil & 57 & 180 & 115 & 280 & 15 & 6 & 24 & 8 & 5 & 16 \\
\hline District & 68 & 150 & 90 & 210 & 10 & 4 & 18 & 6 & 3 & 10 \\
\hline Division & 51 & 105 & 75 & 165 & 5 & 2 & 12 & 6 & 4 & 12 \\
\hline \multicolumn{11}{|l|}{ Active employment } \\
\hline Yes & 87 & 120 & 90 & 195 & 12 & 7 & 21 & 8 & 5 & 14 \\
\hline No & 285 & 240 & 150 & 345 & 18 & 11 & 32 & 12 & 5 & 18 \\
\hline \multicolumn{11}{|l|}{ Lump size (mm) } \\
\hline $1-5$ & 164 & 255 & 120 & 330 & 12 & 5 & 32 & 9 & 4 & 12 \\
\hline $6-10$ & 83 & 210 & 105 & 300 & 9 & 4 & 20 & 8 & 3 & 16 \\
\hline $11-20$ & 81 & 120 & 75 & 205 & 11 & 6 & 21 & 6 & 2 & 11 \\
\hline $21-30$ & 26 & 105 & 60 & 135 & 14 & 6 & 30 & 5 & 2 & 7 \\
\hline $31-50$ & 18 & 45 & 15 & 60 & 18 & 7 & 32 & 3 & 2 & 6 \\
\hline
\end{tabular}

$a_{1}$ US $\$=139.9$ Rs. 


\begin{tabular}{|c|c|c|c|c|c|c|c|c|c|c|}
\hline \multirow[t]{2}{*}{ Characteristic } & \multirow[t]{2}{*}{ No. } & \multicolumn{3}{|c|}{ Physician delay (days) } & \multicolumn{3}{|c|}{ Treatment delay (days) } & \multicolumn{3}{|c|}{ System delay (days) } \\
\hline & & Median & $25^{\text {th }}$ & $75^{\text {th }}$ & Median & $25^{\text {th }}$ & $75^{\text {th }}$ & Median & $25^{\text {th }}$ & $75^{\text {th }}$ \\
\hline Overall & 372 & 24 & 15 & 42 & 26 & 12 & 44 & 30 & 14 & 58 \\
\hline \multicolumn{11}{|l|}{ Age (years) } \\
\hline $11-20$ & 18 & 15 & 9 & 28 & 14 & 5 & 21 & 20 & 8 & 32 \\
\hline $21-30$ & 56 & 16 & 8 & 30 & 16 & 6 & 28 & 28 & 10 & 40 \\
\hline $31-40$ & 84 & 24 & 12 & 40 & 24 & 12 & 40 & 30 & 12 & 54 \\
\hline $41-50$ & 123 & 20 & 11 & 42 & 20 & 7 & 32 & 26 & 11 & 58 \\
\hline $51-60$ & 62 & 20 & 10 & 34 & 26 & 10 & 30 & 24 & 9 & 38 \\
\hline $61-70$ & 29 & 18 & 13 & 28 & 12 & 4 & 21 & 22 & 6 & 30 \\
\hline \multicolumn{11}{|l|}{ Initial symptoms } \\
\hline Painless lump/tumour & 189 & 30 & 10 & 50 & 30 & 13 & 40 & 40 & 12 & 64 \\
\hline Change in breast & 48 & 23 & 8 & 40 & 22 & 7 & 34 & 28 & 8 & 46 \\
\hline Discharge/ bleeding & 33 & 22 & 11 & 41 & 24 & 11 & 38 & 26 & 7 & 42 \\
\hline Lump under arm & 47 & 21 & 8 & 38 & 28 & 12 & 44 & 30 & 10 & 40 \\
\hline Dimpling & 23 & 14 & 6 & 30 & 20 & 7 & 32 & 28 & 8 & 40 \\
\hline Rash & 32 & 20 & 10 & 39 & 26 & 11 & 42 & 22 & 7 & 38 \\
\hline \multicolumn{11}{|l|}{ Menopausal status } \\
\hline Premenopausal & 158 & 27 & 11 & 44 & 30 & 12 & 45 & 30 & 14 & 52 \\
\hline Postmenopausal & 214 & 28 & 10 & 40 & 32 & 15 & 48 & 26 & 16 & 60 \\
\hline \multicolumn{11}{|l|}{ Marital status } \\
\hline Married & 304 & 26 & 15 & 45 & 26 & 12 & 46 & 26 & 12 & 45 \\
\hline Unmarried & 68 & 22 & 10 & 38 & 20 & 8 & 32 & 25 & 11 & 38 \\
\hline \multicolumn{11}{|l|}{ Education } \\
\hline Illiterate & 162 & 23 & 9 & 36 & 29 & 9 & 41 & 36 & 11 & 56 \\
\hline Primary & 43 & 22 & 13 & 40 & 24 & 12 & 40 & 32 & 12 & 54 \\
\hline Middle & 22 & 20 & 10 & 34 & 23 & 9 & 32 & 29 & 10 & 50 \\
\hline Metric & 72 & 24 & 13 & 44 & 25 & 14 & 38 & 34 & 12 & 58 \\
\hline Intermediate & 34 & 24 & 12 & 40 & 20 & 8 & 30 & 26 & 13 & 38 \\
\hline Bachelor & 23 & 18 & 10 & 36 & 16 & 6 & 28 & 20 & 8 & 32 \\
\hline Master & 16 & 16 & 8 & 30 & 12 & 4 & 21 & 16 & 7 & 28 \\
\hline \multicolumn{11}{|c|}{ Household income (Rs/month) a } \\
\hline$<10000$ & 84 & 28 & 16 & 46 & 30 & 12 & 46 & 42 & 12 & 64 \\
\hline $10000-20000$ & 62 & 30 & 12 & 42 & 26 & 12 & 38 & 38 & 10 & 60 \\
\hline $21000-30000$ & 65 & 18 & 9 & 34 & 22 & 8 & 36 & 34 & 14 & 52 \\
\hline $31000-40000$ & 78 & 18 & 8 & 36 & 24 & 10 & 32 & 30 & 12 & 46 \\
\hline $41000-50000$ & 53 & 16 & 9 & 35 & 18 & 7 & 28 & 26 & 10 & 40 \\
\hline$>50000$ & 30 & 14 & 6 & 25 & 16 & 5 & 22 & 18 & 8 & 26 \\
\hline \multicolumn{11}{|l|}{ Area of residence } \\
\hline Village & 103 & 35 & 14 & 58 & 26 & 12 & 48 & 42 & 14 & 62 \\
\hline Town & 93 & 28 & 12 & 45 & 28 & 15 & 46 & 38 & 12 & 56 \\
\hline Tehsil & 57 & 16 & 7 & 28 & 26 & 11 & 40 & 32 & 11 & 52 \\
\hline District & 68 & 12 & 6 & 26 & 22 & 9 & 36 & 28 & 8 & 40 \\
\hline Division & 51 & 10 & 5 & 24 & 16 & 6 & 22 & 22 & 7 & 32 \\
\hline \multicolumn{11}{|l|}{ Active employment } \\
\hline Yes & 87 & 20 & 11 & 35 & 18 & 12 & 30 & 24 & 9 & 38 \\
\hline No & 285 & 26 & 12 & 44 & 24 & 16 & 44 & 36 & 15 & 60 \\
\hline \multicolumn{11}{|l|}{ Lump size (mm) } \\
\hline $1-5$ & 164 & 21 & 8 & 44 & 28 & 12 & 42 & 30 & 12 & 62 \\
\hline $6-10$ & 83 & 17 & 6 & 36 & 22 & 10 & 38 & 32 & 15 & 54 \\
\hline $11-20$ & 81 & 16 & 8 & 32 & 20 & 8 & 32 & 24 & 10 & 42 \\
\hline $21-30$ & 26 & 12 & 5 & 28 & 18 & 8 & 26 & 18 & 10 & 36 \\
\hline $31-50$ & 18 & 8 & 3 & 18 & 12 & 5 & 20 & 10 & 6 & 28 \\
\hline
\end{tabular}

$a_{1}$ US $\$=139.9$ Rs. 


\begin{tabular}{|c|c|c|c|c|}
\hline \multirow[t]{2}{*}{ Characteristic } & \multirow[t]{2}{*}{ No. } & \multicolumn{3}{|c|}{ Total delay (days) } \\
\hline & & Median & $25^{\text {th }}$ & $75^{\text {th }}$ \\
\hline Total & 372 & 310 & 160 & 430 \\
\hline \multicolumn{5}{|l|}{ Age (years) } \\
\hline $11-20$ & 18 & 85 & 40 & 150 \\
\hline $21-30$ & 56 & 120 & 55 & 230 \\
\hline $31-40$ & 84 & 180 & 80 & 310 \\
\hline $41-50$ & 123 & 185 & 90 & 270 \\
\hline $51-60$ & 62 & 210 & 114 & 314 \\
\hline $61-70$ & 29 & 190 & 106 & 270 \\
\hline \multicolumn{5}{|l|}{ Initial symptoms } \\
\hline Painless lump/tumour & 189 & 280 & 140 & 410 \\
\hline Change in breast & 48 & 200 & 80 & 305 \\
\hline Discharge/bleeding & 33 & 205 & 75 & 285 \\
\hline Lump under arm & 47 & 160 & 70 & 240 \\
\hline Dimpling & 23 & 132 & 92 & 206 \\
\hline Rash & 32 & 120 & 63 & 192 \\
\hline \multicolumn{5}{|l|}{ Menopausal status } \\
\hline Premenopausal & 158 & 264 & 150 & 345 \\
\hline Postmenopausal & 214 & 286 & 174 & 330 \\
\hline \multicolumn{5}{|l|}{ Marital status } \\
\hline Married & 304 & 315 & 260 & 416 \\
\hline Unmarried & 68 & 214 & 95 & 324 \\
\hline \multicolumn{5}{|l|}{ Education } \\
\hline Illiterate & 162 & 282 & 163 & 368 \\
\hline Primary & 43 & 270 & 106 & 320 \\
\hline Middle & 22 & 190 & 84 & 276 \\
\hline Metric & 72 & 228 & 127 & 308 \\
\hline Intermediate & 34 & 130 & 73 & 285 \\
\hline Bachelor & 23 & 116 & 68 & 194 \\
\hline Masters & 16 & 96 & 59 & 137 \\
\hline \multicolumn{5}{|c|}{ Household income (Rs/month) ${ }^{a}$} \\
\hline$<10000$ & 84 & 330 & 264 & 412 \\
\hline $10000-20000$ & 62 & 283 & 196 & 365 \\
\hline $21000-30000$ & 65 & 245 & 143 & 336 \\
\hline $31000-40000$ & 78 & 228 & 106 & 312 \\
\hline $41000-50000$ & 53 & 185 & 94 & 278 \\
\hline$>50000$ & 30 & 124 & 65 & 186 \\
\hline \multicolumn{5}{|l|}{ Area of residence } \\
\hline Village & 103 & 276 & 163 & 370 \\
\hline Town & 93 & 243 & 146 & 335 \\
\hline Tehsil & 57 & 184 & 78 & 246 \\
\hline District & 68 & 172 & 65 & 208 \\
\hline Division & 51 & 152 & 60 & 186 \\
\hline \multicolumn{5}{|l|}{ Active employment } \\
\hline Yes & 87 & 178 & 87 & 214 \\
\hline No & 285 & 285 & 194 & 358 \\
\hline \multicolumn{5}{|l|}{ Lump size (mm) } \\
\hline $1-5$ & 164 & 244 & 136 & 332 \\
\hline $6-10$ & 83 & 218 & 145 & 280 \\
\hline $11-20$ & 81 & 160 & 84 & 238 \\
\hline $21-30$ & 26 & 126 & 58 & 173 \\
\hline $31-50$ & 18 & 64 & 42 & 86 \\
\hline
\end{tabular}

${ }_{1}$ US\$ $=139.9$ Rs. 


\begin{tabular}{lcc}
\hline Table 5 Correlation between selected factors and total delay among breast cancer patients $(\boldsymbol{n}=\mathbf{3 7 2})$, Lahore, 2016-2018 \\
Factor & Correlation $(\boldsymbol{r})$ & $\mathbf{P}$-value \\
\hline Area of residence & 0.965 & 0.007 \\
Lump size & 0.897 & 0.039 \\
Income per month & 0.8617 & 0.027 \\
Initial symptoms & 0.847 & 0.036 \\
Education & 0.7197 & 0.068 \\
Age & 0.491 & 0.322 \\
\hline
\end{tabular}

education can significantly reduce delays. Less-educated patients cannot effectively decide about their health issues in a proper time period. Jassem et al. studied delays in 12 countries and noted that higher education was associated with less delay (21). The perception among a population that cancer is incurable and treatment is not available makes the life of breast cancer victims shorter. Both ignorance and illiteracy lead to the presentation of disease at later stages. It is important in developing countries like Pakistan to identify high risk patients in a timely manner and to treat new cases with curative intent.

A cohort study from the United States of America reported financial problems caused self-delay in $17 \%$ of breast cancer patients below 40 years of age (22). Our study shows low income per month is highly correlated with delay in treatment. Low financial status prioritizes health at a low level in comparison with other essentials of everyday life. We found that employed women underwent less delay in comparison with unemployed women. Other research has found employment to be a barrier in the process of diagnosis and treatment of breast cancer (23). Employment is the characteristic of educated and self-dependent women: employed women take their own decisions and face fewer social and cultural barriers. Homemakers are financially and culturally dependent on husbands or family heads and face more delays. Dianatinasab et al. also found that, among Iranian women, employed breast cancer patients faced fewer delays (24).

Our research points out that unmarried women with breast cancer are detected early in comparison to married women. Married women may fear of divorce and remarriage of the husband as in our society cancer is also suspected as being a contiguous disease. So a married woman may decide not to pursue a diagnosis even if she suspects initial symptoms of breast cancer.

A majority of the Pakistani population resides in rural areas. Our study demonstrated that rural breast cancer patients have multiple delays in comparison with those from urban areas. Rural residents have no direct approach to cancer centres and also their awareness level is not good. They also have to travel long distances to approach medical facilities. Rural culture and social values also prohibit women from discussing their disease with others. Other studies from developing countries have reported women living in rural areas encountering long delays $(13,14,18)$. The health care system in Pakistan is not strong enough to cater to patients at grass roots level. Cancer days are observed to increase awareness among the public but their impact is limited.

There is a requirement for continuous awareness programmes to make the population aware about their health concerns. Early detection can reduce the mortality rate and the burden on health facilities: mammography plays a vital role in screening programmes (25). Developing countries have limited resources and the population mostly depends on the government medical care system. In most of the developing countries infrastructure for screening and early detection of breast cancer do not exist even though early detection can decrease the burden of incurable breast cancer patients on the health care facilities. Due to the lack of a social security system, people are reluctant to accept the cost of health facilities in Pakistan; there is no breast cancer screening programme and the cost of mammography is high. An awareness of breast self-examination and making people realize that cancer is curable at the early stages may reduce delays and reduce the mortality rate.

\section{Conclusion}

The women included in this study showed delay in presentation due to lack of resources and lack of awareness about the disease. There is a strong need to create awareness about the disease among women from rural areas and there is need to develop cancer care centres in primary health care departments so that diagnosis of the disease can be made possible at early stage.

\section{Funding: None}

Competing interests: None declared.

\begin{tabular}{lcc}
\hline Table 6 Association between selected factors and total delay among breast cancer patients, Lahore, 2016-2018 & P-value \\
Factor & Chi square & 0.098 \\
Menopause status & 2.731 & $<0.001$ \\
Active employment & 21.588 & $<0.001$ \\
Marital status & 48.917 & \\
\hline
\end{tabular}




\section{Retards de diagnostic et de traitement du cancer du sein liés à de multiples facteurs au Pakistan}

\section{Résumé}

Contexte : Le cancer du sein a le taux d'incidence le plus élevé parmi tous les types de cancer dans le monde. Il existe des preuves solides montrant que le retard de consultation d'un oncologue peut entraîner une diminution de la survie.

Objectifs : La présente étude explore les facteurs causant des retards de diagnostic et de traitement chez les patientes atteintes de cancer du sein admises à l'hôpital Jinnah de Lahore entre 2016 et 2018.

Méthodes : Les données de 372 patientes ont été recueillies ; elles comprenaient notamment les caractéristiques de la tumeur, les premiers symptômes, les connaissances et l'expérience en matière du cancer du sein, la première consultation chez un médecin, etc. Nous avons calculé les intervalles en matière de retard pour le patient, le médecin, le système et les intervalles totaux.

Résultats : Les cas de cancer du sein montraient un retard moyen plus important chez les femmes âgées (plus de 50 ans) que chez les femmes plus jeunes. Les femmes ayant un nodule indolore comme symptôme initial affichaient le retard le plus long avec un total médian de 280 jours $\left(25^{\mathrm{e}}\right.$ et $75^{\mathrm{e}}$ percentiles, 140 et 410 jours respectivement). Les symptômes initiaux étaient corrélés au retard total $(p=0,036)$. Les femmes éduquées montraient un retard de traitement plus court que les femmes analphabètes $(p=0,068)$. Le retard était significatif pour les femmes vivant en milieu rural $(p=0,007)$. La taille du nodule révélait une corrélation avec le retard $(p=0,039)$. Les patients dont le revenu du ménage est faible (<10 000 roupies) présentaient un retard de diagnostic plus important $(p=0,027)$ et les femmes actives affichaient un retard plus court $(p<0,0001)$. Les femmes célibataires étaient diagnostiquées plus tôt que les femmes mariées $(p<0,001)$.

Conclusions : Les femmes tardaient à consulter un médecin en raison du manque de ressources et de sensibilisation à la maladie, de la peur de la chirurgie et de la chimiothérapie. L'utilisation de méthodes de traitement traditionnelles permet de diagnostiquer la maladie à des stades plus avancés.

$$
\begin{aligned}
& \text { تأخر تشخيص سرطان الثدي وعلاجه واقتران ذلك بعوامل متعددة في باكستان } \\
& \text { عمر ان ماجد، رنا أمان الله، عبد الو احد أنور، حافظ رفيق، فايزة عمران } \\
& \text { الخالاصة }
\end{aligned}
$$

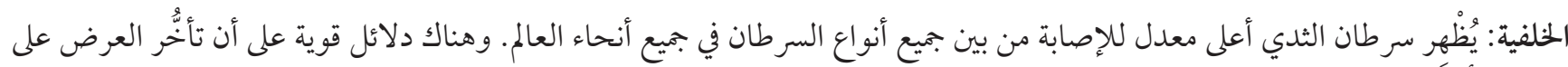

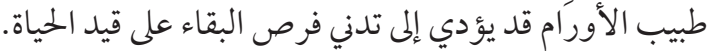

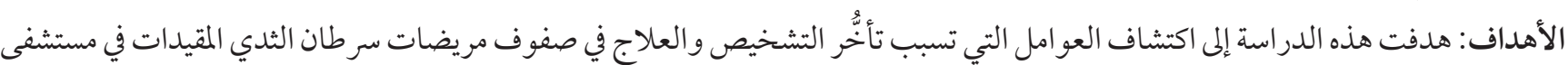

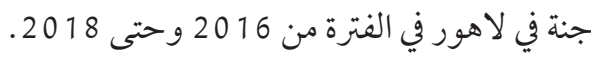

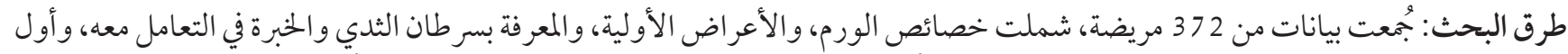

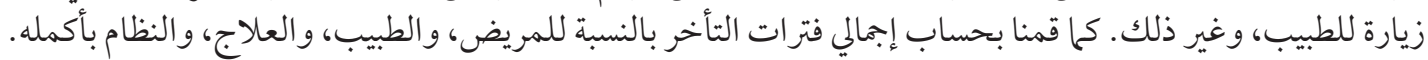

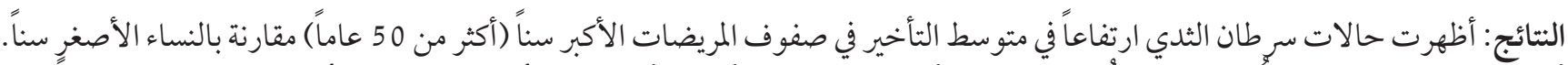

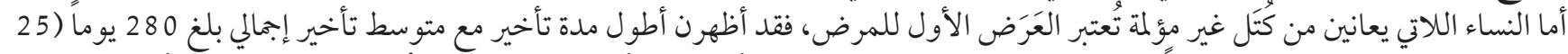

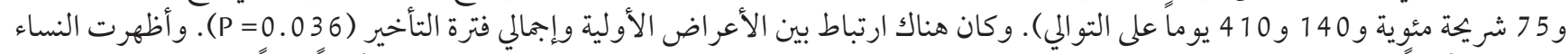

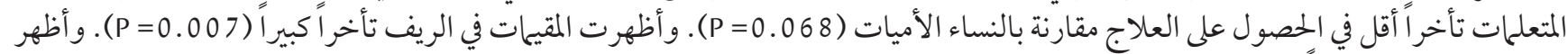

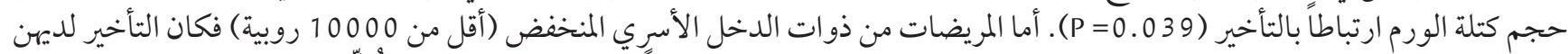

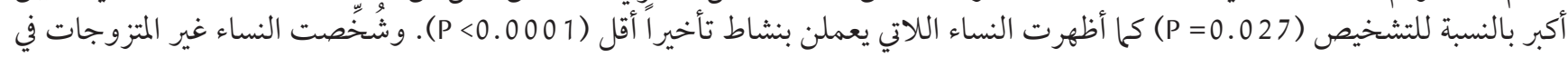

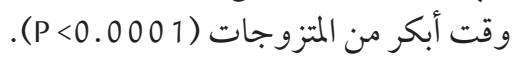

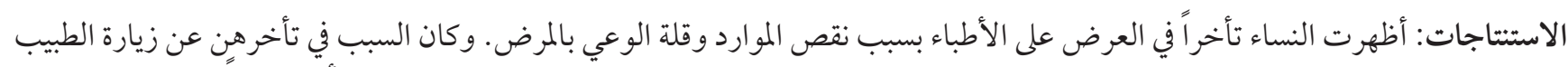

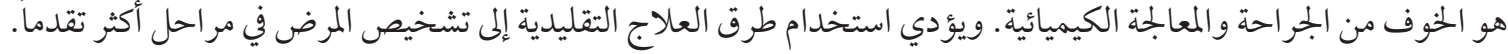




\section{References}

1. Breast cancer facts \& figures 2017-2018. Atlanta: American Cancer Society Inc.; 2017.

2. Bray F, Ferlay J, Soerjomataram I, Siegel RL, Torre LA, Jemal A. Global cancer statistics 2018: GLOBOCAN estimates of incidence and mortality worldwide for 36 cancers in 185 countries. CA Cancer J Clin. 2018 Nov;68(6):394-424. doi:10.3322/caac.21492

3. Majeed I, Rana A, Rafique HM, Waheed Anwar A, Mahmood F. Time delay barriers in diagnosis and treatment of cancer. World Cancer Res J. 2018;5(3):e1118.

4. Shamsi U. Patient delay in breast cancer diagnosis, its associated factors and stage of breast cancer at first presentation. J Global Oncol. 2018;4(Suppl. 2). doi:10.1200/jgo.18.91000

5. Agodirin O, Olatoke SA, Rahman GA, Olaogun J, Kolawole O, Agboola JO, et al. Impact of primary care delay on progression of breast cancer in a black African population: a multicentered survey, J Cancer Epidemiol. 2019;(4):1-10. doi:10.1155/2019/2407138

6. Bonsu AB, Ncama BP. Recognizing and appraising symptoms of breast cancer as a reason for delayed presentation in Ghanaian women: A qualitative study PLoS One. 2019; PLoS One. 2019 Jan 9;14(1):e0208773. doi:10.1371/journal.pone.0208773

7. Rivera-Franco MM, Leon-Rodriguez E. Delays in breast cancer detection and treatment in developing countries. Breast Cancer (Auckl). 2018 Jan 8;12:1178223417752677. doi:10.1177/1178223417752677

8. Parsonage RK, Hiscock J, Law R-J, Neal RD. Patient perspectives on delays in diagnosis and treatment of cancer. Br J Gen Pract. 2017 Jan;67(654):e49-e56. doi:10.3399/bjgp16X688357

9. Ojala K, Meretoja TJ, Mattson J, Salminen-Peltola P, Leutola S, Berggren M, et al. The quality of preoperative diagnostics and surgery and their impact on delays in breast cancer treatment - A population based study. Breast. 2016;26:80-6. doi:10.1016/j. breast.2015.12.009

10. Li Y, Zhou Y, Mao F, Guan J, Lin Y, Wang X, et al. The influence on survival of delay in the treatment initiation of screening detected nonsymptomatic breast cancer. Sci Reports. 2019;9:10158.

11. Gulzar F, Akhtar MS, Sadiq R, Bashir S, Jamil S, Baig SM. Identifying the reasons for delayed presentation of Pakistani breast cancer patients at a tertiary care hospital. Cancer Manag Res. 2019;11:1087-96. doi:10.2147/CMAR.S180388

12. Lopes TCR, Gravena AAF, Demitto M, et al. Delay in diagnosis and treatment of breast cancer among women attending a reference service in Brazil. Asian Pac J Cancer Prev. 2017 Nov 26;18(11):3017-23. doi:10.22034/APJCP.2017.18.11.3017

13. Grosse Frie K, Kamaté B, Traore CB, Ly M, Malle B, Coulibaly B, et al. Factors associated with time to first healthcare visit, diagnosis and treatment, and their impact on survival among breast cancer patients in Mali. PLoS One. 2018;13(11):e0207928. doi:10.1371/journal.pone.0207928

14. Maghous A, Rais F, Ahid S, Benhmidou N, Bellahamou K, Loughlimi H, et al. Factors influencing diagnosis delay of advanced breast cancer in Moroccan women. BMC Cancer. 2016 Jun 7;16:356. doi:10.1186/s12885-016-2394-y

15. Jaiswal K, Hull M, Furniss AL, Doyle R, Gayou N, Bayliss E. J Natl Compr Canc Netw. 2018 Dec;16(12):1451-7. doi:10.6004/jnccn.2018.7067

16. Chavez-MacGregor M, Clarke CA, Lichtensztajn DY, Giordano SH. Delayed initiation of adjuvant chemotherapy among patients with breast cancer. JAMA Oncol. 2016 Mar;2(3):322-9. doi:10.1001/jamaoncol.2015.3856.

17. Hoffman HJ, Khan A, Ajmera KM, Zolfaghari L, Schenfeld JR, Levine PH. Initial response to chemotherapy, not delay in diagnosis, predicts overall survival in inflammatory breast cancer cases. Am J Clin Oncol. 2014 Aug;37(4):315-21. doi:10.1097/ COC.obo13e318271b34b.

18. Setyowibowo H, Sijbrandij M, Iskandarsyah A, Hunfeld JAM, Sadarjoen SS, Badudu DF, et al. A protocol for a cluster-randomized controlled trial of a self-help psychoeducation programme to reduce diagnosis delay in women with breast cancer symptoms in Indonesia. BMC Cancer. 2017;17(1):284. doi:10.1186/s12885-017-3268-7

19. Habibullah S, Haider G, Ashraf J, Dahar SA. To determine the factors responsible for diagnostic delay of breast cancer among women. Pak J Med Res.2016;55(3).

20. Kitano A, Shimizu C, Yamauchi H, Akitani F, Shiota K, Miyoshi Y, et al. Factors associated with treatment delay in women with primary breast cancer who were referred to reproductive specialists. ESMO Open. 2019 Mar 5;4(2):e000459. doi:10.1136/esmoopen-2018-000459

21. Jassem J, Ozmen V, Bacanu F, Drobniene M,et al. Delays in diagnosis and treatment of breast cancer: a multinational analysis, Eur J Public Health. 2014 Oct;24(5):761-7. doi:10.1093/eurpub/ckt131. Epub 2013 Sep 12.

22. Ruddy KJ, Gelber S, Tamimi RM, Schapira L, Come SE, Meyer ME, et al. Breast cancer presentation and diagnostic delays in young women. Cancer. 2014;120(1):20-5. doi:10.1002/cncr.28287

23. Doyle K, Newman RM, Sullivan M, Pergolotti M, Braverman B, Cheville AL. Employment concerns and associated impairments of women living with advanced breast cancer, Arch Rehabil Res Clin Translation. 2019 June;1(1-2):100004. https://doi.org/10.1016/j. arrct.2019.100004

24. Dianatinasab M1, Fararouei M, Mohammadianpanah M, Zare-Bandamiri M. Impact of social and clinical factors on diagnostic delay of breast cancer: a cross-sectional study. Medicine (Baltimore). 2016 Sep;95(38):e4704. doi:10.1097/MD.0000000000004704

25. Seneviratne S, Campbell I, Scott N, Shirley R, Lawrenson R. Impact of mammographic screening on ethnic and socioeconomic inequities in breast cancer stage at diagnosis and survival in New Zealand: a cohort study. BMC Public Health. 2015 Jan 31;15:46. doi:10.1186/s12889-015-1383-4 